\title{
Cryptococcal Meningitis in a tertiary Hospital in Pretoria Mortality and risk factors - a retrospective cohort study
}

\author{
J Hiesgen $^{1}$, C Schutte ${ }^{1}$, S Olorunju ${ }^{2}$, J Retief $^{3}$ \\ ${ }^{1}$ Department of Neurology, Kalafong Hospital, University of Pretoria, Pretoria, South Africa \\ ${ }^{2}$ South African Medical Research Council, Pretoria, South Africa \\ ${ }^{3}$ Department of Internal Medicine, Kalafong Hospital, University of Pretoria, South Africa
}

Corresponding author: J Hiesgen (juliane.hiesgen@up.ac.za)

\begin{abstract}
Aim: This retrospective cohort study analyzes the impact of possible risk factors on the survival chance of patients with Cryptococcal meningitis (CM). These factors include the patient's socioeconomic background, age, gender, presenting symptoms, comorbidities, laboratory findings and, in particular, non-adherence versus adherence to therapy.

Methods: Data was collected from all adult patients admitted to Kalafong Hospital with laboratory confirmed CM over a period of 24 months. We analyzed the data by the presentation of descriptive summary statistics, logistic regression was used to assess factors which showed association between outcome of measure and factor. Furthermore, multivariable logistic regression analysis using all the factors that showed significant association in the cross tabulation was applied to determine which factors had an impact on the patients' mortality risk.
\end{abstract}


Results: 87 patients were identified. All except one were HIV positive, of which 55.2\% were Antiretroviral Therapy (ART) naïve. A history of previous Tuberculosis (TB) was given by 25 patients $(28.7 \%)$ and $49(56.3 \%)$ were on TB treatment at admission or started during their hospital stay. In-hospital mortality was $31 \%$. Statistical analysis showed that ART naïve patients had 9.9 (CI 95\% 1.2-81.2, $\mathrm{p}<0.0032$ ) times greater odds of dying compared to those on ART, with 17 from 48 patients $(35.4 \%)$ dying compared with 1 out of 21 patients $(4.8 \%)$ on treatment. Defaulters had 14.7 (CI 95\% 1.6-131.6, p<0.016) times greater odds of dying, with 9 from 18 patients dying (50\%), compared to the non-defaulters. In addition, patients who presented with nausea and vomiting had a $6.3(95 \%$ CI 1.7-23.1, p<0.005) times greater odds of dying $(18 / 47$, $38.3 \%$ ); this remained significant when adjusted for ART naïve patients and defaulters.

Conclusion: $\mathrm{CM}$ is still a common opportunistic infection in people living with HIV/AIDS resulting in hospitalization and a high mortality. Defaulting ART and presentation with nausea and vomiting were associated with a significantly increased mortality risk.

\section{Introduction}

The number of people living with HIV in South Africa increased to approximately 6.4 million in 2012, resulting in an estimated prevalence of $12.2 \% .^{1}$ Considerable improvements have been achieved through the implementation of the ART enrolment program in South Africa during the last 10 years, with currently about 2.6 million HIV-positive patients on treatment. If ART is started before the CD 4 counts drops to below 200 cells/ $\mu 1$ and if patients do not default their treatment, a dramatic improvement in the life expectancy of patients with HIV/AIDS has been shown. $^{2}$ 
Although AIDS and AIDS-defining opportunistic infections are not notifiable diseases in South Africa, previous data from surveillance projects have shown that patients with cryptococcal meningitis (CM) match the demographics of South Africans living with HIV with respect to age, gender and geographical location. Therefore, and because CM is a common condition which can be accurately diagnosed by laboratory findings, $\mathrm{CM}$ was proposed as an ideal opportunistic infection for monitoring the efficacy of HIV testing and treatment programs in South Africa. A substantial reduction in the incidence of $\mathrm{CM}$ has been expected over the years. ${ }^{3}$

However, despite the largest ART Enrolment Program in the world, clinicians still see a large number of patients with $\mathrm{CM}$ in South African hospitals. Cryptococcal meningitis is the most common life threatening mycosis in people living with HIV/AIDS ${ }^{4}$ and the second most common opportunistic infection after Tuberculosis (TB) in South Africa. ${ }^{5}$ CM is also the most common cause of laboratory confirmed meningitis. ${ }^{3}$ Recent studies confirm the high prevalence of CM in South African Hospitals, a high in-patient mortality and poor long term prognosis. ${ }^{7,8}$ This has been observed despite the availability of good diagnostic tools and updated national guidelines for the diagnosis and treatment of CM in South Africa. ${ }^{9}$

The poor outcome in the treatment of CM patients suggests that possible risk factors regarding their chance of survival are not sufficiently recognized. To address this shortcoming, a retrospective study was conducted to investigate the impact of several risk factors on the chance of survival of patients with CM. These factors include the patient's socio-economic background, age, gender, presenting symptoms, comorbidities, laboratory findings and, in particular, nonadherence versus adherence to therapy. 


\section{Methods}

This study was conducted retrospectively at Kalafong Hospital in Pretoria. Data including patients' socio-economic background, age, gender, presenting symptoms, comorbidities, bloodand CSF results and, in particular, adherence to ART, were collected and analyzed for all adult patients with CM admitted between January 2012 and December 2013 (24 month period). The patients were identified via screening of all discharge summaries from the Department of Internal Medicine, and patient files were then retrieved from the record division at the Hospital. Only patients with confirmed CM (positive CSF diagnosis by India Ink Stain, Cryptococcal Antigen Test (CrAg) and/or fungal culture) were included.

Patients were initially treated with a combination of intravenous Amphotericin B $(1 \mathrm{mg} / \mathrm{kg})$ and Fluconazole $(800 \mathrm{mg}$ ) either orally or intravenously over a period of 14 days. Deviations from the recommended regime, consisting of either absence of one antifungal agent, reduced duration of intensive phase or insufficient dosages, were identified and included in the analyzis.

In cases with multiple admissions socio-economic data was drawn from the initial admission, while all other data - including signs and symptoms, lab results, outcome etc. - were evaluated separately.

Cases with CM Immune Reconstitution Inflammatory Syndrome (IRIS) were identified retrospectively by applying modified criteria suggested by Haddow et al. ${ }^{10}$ in 2010 for the International Network for the study of HIV-associated IRIS (INSHI).

The data was transferred into a data acquisition form and analyzed by the presentation of descriptive summary statistics. This was followed by logistic regression analyzis to assess factors which showed an association (Chi-square test at $\mathrm{p} \leq 0.05$ ) between outcome of measure and 
factor. Furthermore, multivariable logistic regression analysis using all the factors that showed significant association in the cross tabulation was applied to determine which factors had an impact on the patients' mortality risk.

Ethical approval was obtained by the Research Ethics Committee of the Faculty of Health Sciences, University of Pretoria (protocol number 430/2013).

\section{Results}

87 patients were identified, with 9 patients having multiple (2-4) admissions, resulting in 100 total admissions for cryptococcal meningitis in 24 months. All patients had laboratory confirmed CM. Out of this 100 admissions, 99 were confirmed via LP and one patient was diagnosed via postmortem examination, showing disseminated cryptococcosis (macroscopic and microscopic cryptococcosis of brain, lungs, liver, kidneys and pancreas).

Out of the 99 diagnostic lumbar punctures 66 showed positivity for all three diagnostic tests, India Ink Stain, Cryptococcal Antigen (CrAg) test as well as culture for Cryptococcus neoformans. Diagnosis of CM was based on any single positive test. Regarding the sensitivity of the CSF tests performed in our cohort, the CrAg had the highest sensitivity with all examined CSF samples positive, followed by the fungal CSF culture with a very high sensitivity of 0.98 and the India Ink stain with 0.81 (see Table 1). Our laboratory did not offer CrAg titres or quantitative cultures. 
Table 1: Confirmation tests on CSF for CM (out of 99 diagnostic lumbar punctures)

\begin{tabular}{lccc}
\hline & $\begin{array}{c}\text { number of LP's } \\
\text { (test done) }\end{array}$ & $\begin{array}{c}\text { number of } \\
\text { positive tests }\end{array}$ & $\begin{array}{c}\text { Sensitivity in our } \\
\text { cohort* }\end{array}$ \\
\hline India Ink Stain & 98 & 80 & 0.81 \\
CrAg test & 96 & 96 & 1.0 \\
CSF culture pos. & 82 & 80 & 0.98 \\
\hline
\end{tabular}

* CM diagnosis was based on any single positive test

All patients except one (98.9\%) were HIV positive. The only HIV negative patient was a 69 year old female patient with chronic alcohol abuse and a CD 4 count of 115 cells/ $\mu 1$. The gender ratio was $44.8 \%$ female versus $55.2 \%$ male patients.

Table 2: Socio-demographic characteristics of the study population

\begin{tabular}{lrccc}
\hline & $\begin{array}{c}\text { Total } \\
(\mathbf{n = 8 7})\end{array}$ & $\begin{array}{c}\text { Non-Survivors } \\
(\mathbf{n = 2 7})\end{array}$ & $\begin{array}{c}\text { Survivors } \\
(\mathbf{n = 6 0})\end{array}$ & p-value \\
\hline Median age in years (IQR) & $34(13)$ & $37(15)$ & $34(12)$ & $0.36^{\mathrm{a}}$ \\
Male & $48(55.2 \%)$ & $16(59.3 \%)$ & $32(53.3 \%)$ & $0.61^{\mathrm{a}}$ \\
HIV positivity & $86(98.9 \%)$ & $26(96.3 \%)$ & $60(100 \%)$ & $0.12^{\mathrm{a}}$ \\
Employed or on pension & $27(31 \%)$ & $9(33.3 \%)$ & $18(30 \%)$ & $0.78^{\mathrm{a}}$ \\
Pre-ART & $48(55.2 \%)$ & $17(63 \%)$ & $31(51.7 \%)$ & $0.34^{\mathrm{a}}$ \\
On ART & $21(24.1 \%)$ & $1(3.7 \%)$ & $20(33.3 \%)$ & $0.003^{\mathrm{a}}$ \\
ART Defaulter & $18(20.7 \%)$ & $9(33.3 \%)$ & $9(15 \%)$ & $0.05^{\mathrm{a}}$ \\
CD4 count in cells / $\mu 1$ & $52(40-65)$ & $40(26-57)$ & $57(49-74)$ & $0.17^{\mathrm{b}}$ \\
$\quad$ & & &
\end{tabular}

${ }^{\text {a }}$ groups were compared using two sample t-test for proportions

* mean CD4 counts compared by using Mann-Whitney Test 
Forty eight patients (55.2\%) were ART naïve, 21 patients $(24.1 \%)$ were on ART and 18 patients (20.7\%) had admitted to defaulting their antiretroviral therapy (socio-demographic characteristics, Table 2).

Symptoms leading to admission and signs at presentation are listed in Table 3. Headache was the most commonly reported symptom (94 admissions) and meningism the most commonly noted clinical feature on admission (73 admissions).

Table 3: Reported symptoms and clinical findings of our study population

\section{Admissions (n=100)}

$\begin{array}{lr}\text { Reported symptoms: } & \\ \text { Headache } & 94 \\ \text { Seizures } & 12 \\ \text { Confusion } & 29 \\ \text { Weight loss } & 37 \\ \text { Nausea and vomiting } & 49 \\ \text { Focal neurological deficit (reported) } & 11 \\ \text { Psychotic features } & 3\end{array}$

\begin{tabular}{lr}
\hline Clinical findings on admission: & 73 \\
Meningism & 25 \\
Fever & 14 \\
Focal neurological deficit & 0 \\
Coma & 26 \\
Cachexia &
\end{tabular}

The CD4 counts ranged between 2 and 368 cells/ $\mu 1$, with a median CD 4 count of 32 cells/ $\mu 1$ and a mean CD 4 count of 52 cells/ $\mu 1$. The mean value in group of patients who did not survive was low with 40 cells/ $\mu$ l compared to 57 cells/ $\mu 1$ in the survivor group, but this did not reach statistical significance $(\mathrm{p}=0.17$, Table 2$)$. 
CM Immune Reconstitution Inflammatory Syndrome (IRIS) was diagnosed in four patients on ART, all of them classified as unmasking IRIS. All of them survived their hospital stay. We could not identify any cases of paradoxical IRIS.

A history of previous TB was given by 25 patients $(28.7 \%)$ and $49(56.3 \%)$ were on TB treatment at admission or started during their hospital stay. Of the 49 patients with a current diagnosis of TB, 9 had proven TB on Sputum or CSF, 2 of which were GenXpert positive and 7 AFB and culture positive.

Twenty five patients died during their first admission and another two while readmitted, resulting in an in-hospital mortality of $31 \%$.

Seventeen out of 48 ART naïve patients died in hospital, resulting in a $35.4 \%$ mortality of this group compared to only one out of $21(4.8 \%)$ in the group of patients on ART. Statistical analyzis showed that ART naïve patients had a 9.9 (CI 95\% 1.2-81.2, p<0.0032) times greater odds of dying compared to those on ART. Looking at the group of defaulters, 9 out of 18 (50\%) died, compared to the low mortality in the patients adherent to treatment. Defaulters had a 14.7 (CI 95\% 1.6-131.6, p<0.016) times greater odds of dying compared to non-defaulters.

In addition, 18 out of $47(38.3 \%)$ patients presenting with nausea and vomiting on admission died. They had a $6.3(95 \%$ CI 1.7-23.1, p<0.005) times greater odds of dying compared to patients without nausea and vomiting; this remained significant even when adjusted for ART naïve patients and defaulters.

No other reported symptom or clinical sign on admission such as, e.g., focal neurological deficit, cachexia, confusion or meningism, showed a significant association with poor outcome. 
Socio-economic factors such as employment, marriage status, living areas etc. showed no significant association with mortality. Analysis of laboratory findings (blood and CSF results) also failed to identify additional significant risk factors for mortality.

During 38 admissions, several LPs were performed (up to six), mainly to measure the opening pressure and to manage raised intracranial pressure. Quantitative pressures were documented in 23 of these LPs, ranging from 5 to $50 \mathrm{~cm} \mathrm{H}_{2} \mathrm{O}$. Neither the pressure nor the number of LPs did correlate with mortality, but the overall mortality in the group of patients with repeated LPs was at $23.7 \%$ lower than the overall in-hospital mortality of our cohort (31\%).

Data regarding the duration of antiretroviral therapy before admission was obtainable in 32 patients. The mean duration of ARVs was 10.3 months, ranging from 4 days to 6 years. When alalyzing the subgroups, we found a very clear difference between the duration of ART before presentation between patients who died (mean $=28.7$ months, 5 months -6 years, $n=6$ ) compared to patients who survived (mean 6,1 months, 4 days -4 years $n=26$ ), as well as between defaulters (mean 24,05 months, 2 weeks- 6 years, $\mathrm{n}=11$ ) and patients on treatment (mean 2.6 months, 4 days- 2 years, $n=21$ ) was found.

Treatment deviations from the recommended treatment guidelines ${ }^{9}$ occurred in 37 patients. The majority of deviations (28) happened during the first year of our study when we had out-of-stock periods, with Amphotericin B and occasionally Fluconazole being unavailable at our hospital (and in South Africa). Renal dysfunction or electrolyte abnormalities that were not appropriately addressed, e.g. with fluid and electrolyte replacement or dosage adjustments, occurred in 10 patients. These deviations however, did not result in a statistically significant increase in mortality of these patients. 


\section{Discussion}

Over a 2 year period we counted 100 admissions for cryptococcal meningitis, representing approximately $0.9 \%$ of all (11495) admissions to the Department of Internal Medicine at Kalafong Hospital during this time. The in-hospital mortality rate was at $31 \%$ very similar to the rate of $30 \%$ reported 2014 from a district hospital in the Eastern Cape, South Africa ${ }^{8}$ and slightly less than the $40.5 \%$ reported from a rural area in KwaZulu Natal in $2011 .{ }^{7}$ Looking at assumed 3 months mortality rates of high income regions of only $9 \%$, it is evident that our inhospital mortality rate is more than three times higher. ${ }^{11}$

A recent retrospective study from India investigated risk factors for fatal outcome in HIV positive patients with CM over a period of about 10 years $^{12}$. The investigators found 91 patients with CM; the mortality at the end point was $40.6 \%$ with a significant difference in the CD4 counts of individuals who died compared to survivors, especially in the groups of patients with a CD 4 count of $<50$ cells $/ \mu 1$ and between 50 and 100 cells/ $\mu 1$. In the paper of Majumder et al. ${ }^{13}$ from 2011, the authors looked at the mortality of AIDS-related CM in 30 consecutive cases. They found a difference in the mean CD4 counts between survivors and non-survivors (88.8 versus 44.4) but this did not reach statistical significance. The mortality was $36.7 \%$ with 10 patients dying during the hospital stay and one during a relapse 7 weeks later. In our cohort, a low CD count did not result in a statistical significant higher mortality. This might be due to either the relatively small number of patients in our study, or to the very low median and mean CD4 count in our cohort. Other authors found that clinically, a low GCS (Glasgow Coma scale) score, evidence of papilledema and an elevated CSF opening pressure on lumbar puncture were identified as poor prognostic factors. ${ }^{13,14}$ In addition a low body mass index (BMI) has been shown previously to be an independent predictor of poor outcome. ${ }^{15,16}$ 
As stated before, we did find a significantly higher mortality in patients presenting with nausea and vomiting. These symptoms could be indicative of a raised intracranial pressure, therefore confirming the findings of the above mentioned studies. However, it is possible that some patients had nausea and vomiting secondary to gastro-enteral disease or other causes such as side effects from current medication (e.g. TB drugs) and not to elevated intracranial pressure. Additional involvement of other organ systems could thus also contribute to a higher mortality. Unfortunately, since ours was a retrospective study, CSF pressure was often not recorded in the files and the data could not be used for analysis. However, there was a trend towards a lower mortality in patients receiving repeated lumbar punctures for pressure management without achieving statistical significance. This could indicate that too few LPs were done in our cohort and underlines the importance of a stringent monitoring and of CSF pressures in all patients at presentation and the accurate treatment of high pressures if present.

The finding that ART defaulters had a much higher risk to die compared to ART naïve patients was neither expected nor found in any other study regarding CM mortality. As the CD4 count did not directly correlate with poor outcome, other reasons than low CD4 counts should be sought to explain the poor outcomes. A meta-analysis of 17 studies, 16 from sub-Saharan Africa showed mortalities for patients lost to follow up ranging from $12 \%$ to $87 \%$, with a combined mortality of $46 \% .{ }^{17}$ McGuire et al. looked at mortality rates of ART naïve HIV-positive patients and defaulters in Malawi in $2010^{18}$ and found a higher mortality rate in defaulters at $54 \%$ versus 49\%. Reasons for defaulting are found to be stigma, care dissatisfaction, poor understanding of the disease, financial challenges and high transport costs as well as improved health and drug side effects ${ }^{17,18,19}$. The cause of the higher mortality in defaulters, however, is not fully clear. Since patients who default treatment share the very low CD4 counts with the pre-ART patients, 
the defaulters might have a longer duration of the disease, as supported by our data, or a more complex deranged immune system with more comorbidities and co-infections.

In our patient cohort the $\mathrm{TB}$ co-infection rate was very high at $56.3 \%$. Therefore the necessity often arises to combine antifungal, anti-mycobacterial and antiretroviral therapy, frequently added to other antibiotic and chronic co-medication. This makes a careful monitoring of not only the clinical condition of the patients but also the liver and renal function essential. In addition to national treatment guidelines, we suggest hospital or departmental guidelines to assist with early diagnosis, monitoring during treatment and adequate response when side effects or complications occur.

CM Immune Reconstitution Inflammatory Syndrome (IRIS) can be assumed if the cryptococcal meningitis occurs after rapid reversal of immunodeficiency due to ART in a patient who was either not diagnosed with cryptococcal disease before, unmasking IRIS, or in a patient who had the disease before and received effective antifungal treatment and secondary prophylaxis, paradoxical IRIS. Unmasking IRIS has to be differentiated from progression of occult cryptococcosis in the context of persisting immune deficiency. Persisting immune deficiency was the main reason to reject the diagnosis of unmasking IRIS in our cohort. Paradoxical IRIS must be distinguished from persistent infection or relapse. We only identified four patients with unmasking IRIS and no case with paradoxical IRIS. We were restricted with our ability to identify IRIS by the fact that baseline HIV VL are not required for initiation of ART and these are not routinely performed during admission. The low mortality in the ART group however, argues against a significant number of unidentified IRIS cases amongst them. 
Previous TB was presumed if patients reported TB treatment in the past. Current TB treatment included patients admitted whilst on TB treatment, which had often been initiated by other health care providers, or had been started in our Hospital. Only 18.4\% (9/49) of patients had microbiologically proven $\mathrm{TB}$, implying in the remaining cases, that the robustness of the diagnosis was either unknown or the patient was empirically treated for TB due to a high index of suspicion. Even in a high TB prevalence country like South Africa it is likely that we included TB negative patients due to the clinical and radiological overlap of these two diseases and the limited yield for microbiological proof of TB. Since the implementation of GeneXpert MTB/Rif technology nationwide in 2014, our ability to diagnose TB accurately and timeously has improved substantially.

There are other limitations to our study which are, firstly, attributable to the retrospective character of our analyzis and, secondly, to an environment with very limited resources and high burden of disease. Opening pressures were not always measured and spinal manometers were not available. Adjusted iv-drip systems and tape rules were used to measure CSF pressure, thereby limiting the accuracy. Although we could retrieve all files, data was sometimes incomplete or missing and because the sample size was relatively small, we may also have overlooked significant associations which a bigger study might have picked up.

\section{Conclusion}

Cryptococcal meningitis is still a common opportunistic infection in South African HIV-positive patients. In our setting, about one third of patients admitted with CM die during their hospital stay. It is therefore essential that efforts to identify and treat patients at risk for CM via screening 
for serum cryptococcal antigen in HIV infected individuals with CD 4 counts less than 100 cells/ $\mu 1$ are stepped up. ${ }^{9} \quad$ Attempts to strengthen our antifungal drug arsenal ${ }^{20}$ must be supported by all clinicians involved in the care of people living with HIV/AIDS.

Two main findings emerged from our study. Firstly, patients presenting with nausea and vomiting had a higher risk to die from CM than patients without these symptoms. We would conjecture that the reason for this finding is a raised intracranial pressure in many of these patients. Thus, special attention should be given to the early identification and immediate management of raised intracranial pressure in all patients with $\mathrm{CM}$.

Secondly, patients who had stopped ART had a much greater risk for fatal outcome than nondefaulters. The reason for this remains unclear but longer duration of the immune suppressive state might play a role. In view of the high defaulter rates among recent cohorts in South Africa, this is of grave concern and health providers should warn patients of the dangers of stopping their antiretroviral medication.

\section{Acknowledgements:}

In completing this paper, the authors are thankful to the referees for constructive comments and to Alex Zimper for critical review of the manuscript.

\section{References}

1. Shisana O, Rehle T, Simbayi LC, Zuma K, Jooste S, Zungu N, Labadarios D, Onoya D et al. South African National HIV prevalence, incidence and behavior survey. 2012. Cape Town. HSRC press 
2. Johnson LF, Mossong J, Dorrington RE et al. Life expectancies of South African adults starting antiretroviral treatment: Collaborative analysis of cohort studies. PLoS Med 10(4):e1001418. doi:10.1371/journal.pmed.1001418

3. McCarthy KM, Cohen c, Schneider H et al. Cryptococcosis in Gauteng: Implications for monitoring of HIV treatment programmes. SAMJ, 2008, 98, 452-454

4. Bicanic T, Harrison T. Cryptococcal meningitis. Br Med Bull, 2005, 72: 99-118

5. Jarvis JN, Harrison TS, Corbett EL, Wood R, Lawn SD. Is HIV-associated tuberculosis a risk factor for development of cryptococcal disease? AIDS, 2010; 24(4):612-614

6. Jarvis JN, Meintjes G, Williams A, Brown Y, Crede T, Harrison TS. Adult meningitis in a setting of high HIV and TB prevalence: findings from 4961 suspected cases. BMC Infect Dis, 2010; 10:67

7. Lessels RJ, Mutevedzi P, Heller T, Newell ML. Poor long-term outcomes for cryptococcal meningitis in rural South Africa. SAMJ, 2011, 101, 251-252

8. Sogbanmu OO, John MA, Lalloo U. Management of cryptococcal meningitis in adults at Mthata Hospital Complex, Eastern cape, South Africa. S Afr J HIVMed, 2014; 15(3): 104107

9. Southern African HIV Clinician Society. The Guideline for the prevention, Diagnosis and Management of Cryptococcal Meningitis among HIV-infected persons: 2013 update. S Afr J HIV Med, 2013; 2 (4):76-86. doi:10.7196/SAJHIVMED.930

10. Haddow LJ, Colebunders R, Meintjes G et al. Cryptococcal immune reconstitution inflammatory syndrome I HIV-1-infected individuals: proposed clinical case definitions. Lancet Infect Dis, 2010;10:791-802

11. Park BJ et al: Estimation of the current global burden of cryptococcal meningitis among persons living with HIV/AIDS, AIDS, 2009, 23: 525-530

12. Sachdeva RK, Randev S, Sharma A et al. A retrospective study of AIDS-associated cryptomeningitis. AIDS Res Hum Retroviruses, 2012 (10): 1020-6

13. Majumder S, Mandal SK, Bandyopadhyay D. Prognostic markers in AIDS-related cryptococcal meningitis. J Assoc Physicians India, 2011, 59:152-4

14. De Vedia L, Arechavala A, Calderon A. et al. Relevance of intracranial hypertension control in the management of Cryptococcus neoformans meningitis related to AIDS. Infection, 2013, Oct. 14, (Epub ahead of print) 
15. Kendi C, Penner J, Koech J et al. Predictors of outcome in routine care for Cryptococcal meningitis in Western Kenya: lessons for HIV outpatient care in resource-limited settings. Postgrad Med J, 2013, 89 (1048): 73-7

16. Anekthananon T, Manosuthi W, Chetchotisakd P et al. Predictors of por clinical outcome of cryptococcal meningitis in HIV-infected patients. Int J STD AIDS, 2011, 22 (11):665-670. doi: 10.1258/ijsa.2011.010538

17. Brinkhof MWG, Pujades-Rodriguez M, Egger M. Mortality of Patients Lost to Follow-up in Antiretroviral Treatment Programmes in resource-Limited Settings: Systematic Review and Meta-Analysis. PLoS ONE ,2009, 4(6): e5790. doi:10.1371/journal.pone.0005790

18. McGuire M, Munyenyembe T, Szumilin E, Heinzelmann A, Le Paih M, Bouithy N, PujadesRodriguez M. Vital State of pre-ART and ART patients defaulting from care in rural Malawi. Trop Med Int Health, 2010, 15: 55-62. doi: 10.1111/j.1365-3156.2010.02504.X

19. Miller CM, Ketlhapile M, Rybasack-Smith H, Rosen S. Why are antiretroviral treatment patients lost to follow up? A qualitative study from South Africa. Trop Med Int Health, 2010, 15: 1:48-54. doi: 10.1111/j.1365-3156.2010.02514.x.

20. Loyse A, Thangaray H, Easterbrook P et al. Cryptococcal meningitis: improving access to essential antifungal medicines in resource-poor countries. Lancet Infect Dis, 2013, 13:629637. doi: 10.1016/S1473-3099(13)70078-1 